\title{
Uso da técnica de busca em vizinhança de grande porte para a programação da escala de motoristas de ônibus urbano
}

\author{
Gustavo Peixoto Silva'; Claudio Barbieri da Cunha ${ }^{2}$
}

\begin{abstract}
Resumo: Este artigo apresenta uma nova abordagem para a resolução do Problema de Programação de Tripulações no Sistema de Transporte Público (PPT). O modelo se baseia na metaheurística GRASP cuja busca local é realizada pelo método da Busca em Vizinhança de Grande Porte, conhecida na literatura como Very Large-Scale Neighborhood Search. O grande diferencial da aplicação desta técnica de busca para o PPT é que, além de incorporar os movimentos de realocação e troca de tarefas, realizados tradicionalmente, ela também permite considerar trocas do tipo 3-optimal, 4-optimal, até o limite de $n$-optimal, para uma solução com $n$ tripulações. A implementação da heurística proposta foi testada com dados de problemas reais de uma empresa que opera em Belo Horizonte, e os resultados foram comparados com as soluções adotadas pela empresa. Desta forma foi possível observar que o modelo apresentado neste trabalho produziu soluções mais econômicas do que aquelas praticadas pela empresa.
\end{abstract}

\begin{abstract}
This paper presents a new approach to solve the Crew Scheduling Problem (CSP) for public mass transport system. The proposed model is based on the GRASP metaheuristic framework, where the local search is performed by the Very Large-Scale Neighborhood (VLSN) search technique. The great differential of this search technique applied to the CSP is that, in addition to task reassigning and swapping movements, adopted in previous work, it also allows considering 3-optimal, 4-optimal, up to $n$-optimal task movements, for a solution with $n$ crews, yielding to improved solutions. The proposed heuristic was tested with data from real problems of a bus company operating in the city of Belo Horizonte, and the results compared to the manual solution adopted by the company. Thus it was observed that the model presented in this work have produced more economical solutions than those used by the company.
\end{abstract}

\section{INTRODUÇÃO}

O planejamento operacional consiste de um conjunto de atividades desenvolvidas pelas empresas gestoras, que têm como objetivo organizar a operação do sistema de transporte coletivo, visando a melhor alocação dos recursos necessários ao atendimento adequado da demanda e à produção dos serviços com a maior eficiência e eficácia possível. Os serviços correspondem à realização das viagens previstas para cada uma das linhas que compõem o sistema. As linhas se caracterizam pelas suas rotas e suas tabelas com os horários de partida de cada viagem, tendo como base seus respectivos pontos iniciais, também ditos pontos de controle da linha. Os principais recursos envolvidos na operação são os veículos e suas tripulações, compostas por motoristas e cobradores, quando for o caso.

$\mathrm{Na}$ programação da frota deve-se determinar a quantidade de veículos necessária à operação da linha ou do conjunto de linhas de cada empresa. São de fundamental importância para otimizar o serviço a definição das características físicas adequadas ao perfil da demanda e do tipo de veículo mais indicado. Normalmente as linhas são divididas por tipo de veículo, o que leva a um problema de alocação de veículos

\footnotetext{
${ }^{1}$ Gustavo Peixoto Silva, Instituto de Ciências Exatas e Biológicas, Universidade Federal de Ouro Preto, Ouro Preto, MG, Brasil. (email: gustavo@iceb.ufop.br).

${ }^{2}$ Claudio Barbieri da Cunha, Escola Politécnica, Universidade de São Paulo, São Paulo, SP, Brasil. (e-mail: cbcunha@usp.br).

Manuscrito recebido em 3/10/2009 e aprovado para publicação em 6/2/2010. Este artigo é parte de TRANSPORTES, volume XVIII, número 2, junho de 2010. ISSN: 2237-1346 (online).
}

com frota homogênea.

A programação da escala das tripulações pode ser diária, semanal, mensal ou até anual, considerando as folgas, descansos, férias, treinamentos e outras atividades da tripulação. Uma programação que apresenta seus serviços equilibrados concorre significativamente para a eficiência do sistema. Assim, uma programação racional deve buscar uma alocação ótima da mão-deobra, considerando o número mínimo de tripulações e de horas extras trabalhadas, de forma a minimizar os custos envolvidos com a remuneração da escala da tripulação. Muitas vezes, a utilização de horas extras, embora mais caras que as horas normais de trabalho, pode ser mais vantajosa do que a contratação de novos profissionais. De qualquer maneira, deve haver um equacionamento destes custos para não incorrer em gastos excessivos com os operadores.

A programação dos veículos e a programação das tripulações são problemas subseqüentes, sendo que a definição da frota em operação, conjuntamente com as regras operacionais e a legislação trabalhista do setor, compõem os dados de entrada para a programação das tripulações. Nesse contexto, a parcela de maior peso nos custos se deve à remuneração das tripulações. Desta forma, torna-se fundamental definir uma programação para a tripulação com custo mínimo, aliviando pressões sobre o aumento nas tarifas do sistema. Essa programação deve ser equilibrada, promovendo condições justas e saudáveis no ambiente de trabalho.

O problema de geração de escalas, denominado na literatura de Problema de Programação de Tripulações 
(PPT) é do tipo NP-Difícil, para o qual não existe algoritmo capaz de encontrar a solução ótima em um tempo de processamento razoável. O problema tem sido largamente estudado e as abordagens exatas mais exploradas utilizam o modelo de recobrimento ou de particionamento de conjuntos. Para resolver tais modelos são empregadas as técnicas de seleção de colunas (Smith e Wren, 1988; Silva et al., 2007), de geração de colunas (Desrochers e Soumis, 1989; Desrochers et al., 1992; Fores et al., 1999), assim como as técnicas de busca em árvore com estratégia do tipo branch-and-price (Barnhart et al., 1998) e branchand-cut (Friberg e Haase, 1999) associada à geração de colunas. A técnica de relaxação Lagrangeana também é utilizada para resolver o problema, como em Freling et al. (2001). Por serem modelos exatos, tais estratégias se limitam a resolver problemas de pequena dimensão e, portanto, não se aplicam aos casos práticos, os quais contam com um grande número de viagens e veículos.

Trabalhos mais recentes apresentam abordagens heurísticas para resolver o problema de programação de tripulações. Com a utilização de métodos heurísticos torna-se possível modelar e resolver problemas de grande porte considerando todas as suas restrições práticas. Dentre as heurísticas utilizadas para resolver o problema pode-se destacar os Algoritmos Genéticos (Clement e Wren, 1995; Wren e Wren, 1995; Wren et al., 2000; Lourenço et al., 2001), a Busca Tabu (Shen e Kwan, 2001; Marinho et al., 2004; Souza et al., 2004), a heurística Simulated Annealing (Silva et al., 2002) e o Método de Busca em Vizinhança Variável VNS (Souza et al., 2004). Com exceção dos algoritmos genéticos, as demais heurísticas realizam basicamente movimentos de realocação e de troca 1 a 1 , ou 2-optimal dos elementos que compõem a solução no processo de busca na sua vizinhança.

Neste trabalho, é apresentado um modelo voltado para a resolução do problema de programação das tripulações baseados na heurística GRASP, cuja busca local é realizada pela técnica de Busca em Vizinhança de Grande Porte (BVGP), proposto por Ahuja et al. (2000). Com esta técnica, podem ser realizados movimentos de realocação e de troca 2-optimal, assim como movimentos de troca 3-optimal, 4-optimal, até o limite de $n$-optimal, onde $n$ representa o número de tripulantes que compõem a escala. Desta forma, é possível explorar um espaço de soluções muito mais complexo do que aqueles pesquisados nas heurísticas apresentadas na literatura. O modelo foi implementado e testado com dados reais de uma empresa que opera na cidade de Belo Horizonte (MG) e os resultados foram comparados com as soluções adotadas pela empresa.

O trabalho está organizado da seguinte maneira: na seção a seguir são apresentadas as características do problema, assim como o modelo de particionamento para o PPT. Na seção 3 é apresentada a técnica de busca em vizinhança de grande porte aplicada ao problema abordado. Nesta seção são apresentados os elementos necessários para aplicar a metaheurística GRASP, detalhada na seção seguinte. Na seção 5 são apresentados os resultados obtidos com o estudo de um caso real. Finalmente, são apresentadas as conclusões do trabalho na seção 6 .

\section{MODELO DE PARTICIONAMENTO DE CONJUNTOS PARA O PPT}

A complexidade computacional do PPT, de natureza combinatória, em que o esforço computacional para sua resolução cresce exponencialmente com o tamanho do problema, torna impossível a obtenção de soluções ótimas do ponto de vista matemático, requerendo o uso de heurísticas, em particular para problemas reais. Assim, tendo em vista a motivação de aplicação a problemas reais do transporte coletivo urbano de passageiros por ônibus encontrados na prática no Brasil, propõe-se uma estratégia de solução heurística que se baseia em um modelo de particionamento de conjuntos, para a resolução do PPT.

Estratégias de solução heurísticas apóiam-se, em geral, em alguma abordagem intuitiva, na qual a estrutura particular do problema pode ser considerada e explorada de forma inteligente, a fim de se obter uma solução satisfatória, levando em consideração o compromisso qualidade versus esforço computacional para a sua obtenção (Silver, 2004). Um dos grandes desafios científicos é o desenvolvimento de estratégias de solução mais simples, mais rápidas e mais robustas, que resultem bom desempenho computacional. Mais especificamente, esse modelo de particionamento de conjuntos define um forma como o problema pode ser particionado e dividido, de forma a possibilitar a aplicação da heurística GRASP (Feo e Resende, 1995), em conjunto com a melhoria das diversas soluções iniciais geradas através do método de Busca em Vizinhança de Grande Porte (Ahuja et al., 2000).

A programação de tripulações tem como dados de entrada a programação dos veículos previamente definida, assim como as regras da empresa e a legislação vigente. A programação dos veículos define os blocos dos veículos, ou seja, o conjunto das viagens a serem executadas por cada veículo da frota. Para resolver o PPT, inicialmente as viagens dos veículos são agrupadas em tarefas. Uma tarefa é uma seqüência de viagens de um mesmo veículo que deve ser executada pela mesma tripulação, por não haver condições operacionais para realizar a troca de tripulações entre as viagens. 
Para realizar a troca de tripulações, ou seja, o "rendimento" de uma tripulação por outra, é necessário que haja uma oportunidade de troca (OT) entre duas viagens. Ocorrerá uma OT sempre que o intervalo de tempo entre o final de uma viagem e o início da viagem subseqüente for superior a um dado valor previamente definido, em um local apropriado para que seja efetuada a troca. As viagens que constituem uma tarefa possuem as seguintes características: i) o tempo de terminal entre as viagens é sempre menor ou igual à OT, e ii) o início e o final de cada tarefa sempre ocorrem nos pontos onde existe um fiscal da empresa ou na garagem. Ao final dessa etapa, todas as viagens sob responsabilidade da empresa são transformadas em tarefas.

Neste trabalho, são considerados os tipos de jornadas, assim com o as restrições trabalhistas contidas no acordo coletivo firmado entre o Sindicato das Empresas de Transporte de Passageiros de Belo Horizonte SETRABH e o Sindicato dos Trabalhadores em Transporte Rodoviários de Belo Horizonte STTRBH, listados a seguir.

- jornada normal de trabalho, ou pegada simples: tem duração de 6 horas e 40 minutos e uma folga para descanso/alimentação de no mínimo 20 minutos, podendo ser fracionada ao longo da jornada;

- jornada do tipo dupla pegada: ocorre quando a tripulação executa um conjunto de tarefas, tem uma folga corrida de pelo menos 2 horas e retorna para cumprir um outro conjunto de tarefas. Neste caso, a folga de 2 horas não é remunerada e não há obrigatoriedade de conter a folga para descanso/alimentação;

- tempo máximo de 2 horas extras por jornada diária;

- tempo mínimo de 11 horas entre o final da jornada e seu início no dia seguinte.

Uma solução do problema de programação de tripulações pode ser vista como um particionamento factível das tarefas a serem executadas pelas diferentes tripulações (ou duplas) em atividade. Este particionamento deve ser viável, sendo que cada partição deve satisfazer as restrições do problema e tem como objetivo utilizar o menor número de duplas, enquanto minimiza o tempo total de horas extras contidas na escala. Portanto, cada partição corresponde a um conjunto de tarefas a serem executadas por uma dada dupla, chamada de jornada diária de trabalho da dupla. O conjunto de todas as jornadas de trabalho das duplas se constitui em uma escala para as tripulações da empresa.

Para formular o PPT como um problema de particionamento, considere $\boldsymbol{T}=\left\{t_{1}, t_{2}, t_{3}, \ldots, t_{n}\right\}$ um con- junto de $n$ tarefas provenientes do agrupamento das viagens contidas nos blocos dos veículos da frota operada por uma determinada empresa de transporte público. Os subconjuntos $D_{1}, D_{2}, D_{3}, \ldots, D_{P}$ podem ser vistos como uma escala com $P$ duplas e definem uma partição factível de $\boldsymbol{T}$ se os mesmos são viáveis, disjuntos e a sua união resulta em $\boldsymbol{T}$. Essa partição pode ser representada como $\boldsymbol{D}=\left\{D_{1}, D_{2}, D_{3}, \ldots, D_{P}\right\}$. Seja $c_{k}\left(D_{k}\right)$ o custo do subconjunto $D_{k}$, calculado em função da remuneração fixa e das horas extras da jornada da dupla $k$. Assim, o problema de particionamento é formalizado a seguir.

$$
\operatorname{Minimiza} c(D)=\sum_{k=1}^{P} c_{k}\left(D_{k}\right)
$$

tal que, $D=\left(D_{1}, D_{2}, D_{3}, \ldots, D_{P}\right)$ é uma partição de $T$.

O problema de particionamento (1) segue a premissa necessária para aplicar a BVGP uma vez que o custo $c_{k}\left(D_{k}\right)$ não depende da maneira como os demais elementos de $\boldsymbol{D}$ são alocados aos respectivos subconjuntos, nem mesmo de como estes subconjuntos são configurados. Isso ocorre para o caso do PPT, uma vez que as tarefas alocadas a cada uma das $D$ duplas definem a ordem ou seqüência em que as mesmas são executadas, assim como os eventuais tempos ociosos, de maneira independente das demais duplas da escala.

\section{O ALGORITMO BVGP PARA O PROBLEMA DE ESCALA DE TRIPULAÇÕES}

A seguir são apresentadas as adaptações que possibilitaram a utilização do método de busca em vizinhança de grande porte (very large-scale neighborhood search) para resolver o PPT. Esse método de busca local foi incorporado à heurística GRASP para resolver o PPT relacionado a empresas de pequeno, médio e de grande porte. Maiores detalhes sobre o método BVGP, que permite explorar vizinhanças com um número muito grande de soluções adjacentes, através da utilização de modelos de fluxo em rede, podem ser encontrados em Ahuja et al. (2000, 2002).

\subsection{Estrutura de vizinhança de troca cíclica}

Uma solução para o PPT só pode ser melhorada com a realocação e/ou troca das tarefas entre as $P$ duplas da escala. Logo, um método de busca em vizinhança pode se basear na realocação ou em trocas aos pares, na qual duas tarefas são trocadas entre as duas tripulações, às quais pertencem. Uma troca cíclica para o PPT pode ser definida por uma sequência de tarefas $t_{1^{-}}$ $t_{2}-t_{3}-\ldots-t_{r}-t_{1}$, sendo que as tarefas $t_{1}, t_{2}, t_{3}, \ldots, t_{r}$ pertencem a diferentes tripulações. Seja $D\left[t_{k}\right]$ a tripulação à qual pertence a tarefa $t_{k}$, então a troca cíclica $t_{1}-t_{2}-t_{3^{-}}$ $\ldots-t_{r}-t_{l}$ representa as seguintes alterações: a tarefa $t_{l}$ é movida da tripulação $D\left[t_{l}\right]$ para a tripulação $D\left[t_{2}\right]$, a 
tarefa $t_{2}$ de $D\left[t_{2}\right]$ para $D\left[t_{3}\right]$, e assim sucessivamente. Finalmente, a tarefa $t_{r}$ é movida de $D\left[t_{r}\right]$ para $D\left[t_{1}\right]$.

Para uma dada partição factível $\boldsymbol{D}$, definimos uma outra partição $\boldsymbol{D}^{\prime}$ como vizinha de $\boldsymbol{D}$ se: (i) $\boldsymbol{D}^{\prime}$ é uma partição factível e (ii) $\boldsymbol{D}^{\prime}$ pode ser obtida a partir de $\boldsymbol{D}$, realizando um movimento de troca cíclica. Definimos uma vizinhança $\boldsymbol{V}(\boldsymbol{D})$ como sendo a coleção de todas as partições que são vizinhas de $\boldsymbol{D}$. Para examinar implicitamente esta vizinhança, foi utilizado o grafo de melhoria associado ao PPT. Esta vizinhança cresce exponencialmente com $P$. Portanto, examiná-la completamente para identificar uma troca cíclica que melhore o custo da solução é ineficiente do ponto de vista computacional. Entretanto, podemos utilizar o conceito de grafo de melhoria, descrito a seguir, para realizar implicitamente a pesquisa na vizinhança.

\subsection{Grafo de melhoria para o PPT}

Um grafo de melhoria (improvement graph) para uma vizinhança com múltiplas trocas é definido para uma partição viável $\boldsymbol{D}$, sendo representado por $G(\boldsymbol{D})$. Para construir o grafo de melhoria que permite aplicar o método BVGP ao PPT, considere $\boldsymbol{T}$ o conjunto das $n$ tarefas, uma partição $\boldsymbol{D}$ das tarefas e, seja $\bar{D}=\left\{d_{1}, d_{2}, \ldots, d_{P}\right\}$, o conjunto de nós que representam as $P$ tripulações (ou duplas) na solução $\boldsymbol{D}$. Então o grafo $G(\boldsymbol{D})=(\boldsymbol{N}, \boldsymbol{E})$ tem o conjunto de nós dado por $\boldsymbol{N}=\boldsymbol{T} \cup \bar{D} \cup\{s\}$, ou seja, um nó para cada tarefa, um nó para cada dupla e um nó extra, dito supernó s. Os arcos do conjunto $\boldsymbol{E}$ são dos seguintes tipos:

a. ligando duas tarefas $\left(t_{i}, t_{j}\right)$, que representa a substituição da tarefa $j$ pela tarefa $i$ na dupla que contém a tarefa $j$. O custo deste tipo de arco é dado por $c\left(\left\{t_{i}\right\} \cup D\left[t_{j}\right] \backslash\left\{t_{j}\right\}\right)-c\left(D\left[t_{j}\right]\right)$;

b. ligando uma tarefa a um dupla $\left(t_{i}, d_{j}\right)$, que representa a inclusão da tarefa $i$ na dupla $j$ sem que qualquer tarefa seja retirada da dupla $j$. Neste caso, o custo do arco é calculado pela expressão $c\left(\left\{t_{i}\right\} \cup D_{j}\right)-c\left(D_{j}\right)$;

c. ligando o super-nó s a cada tarefa $\left(s, t_{j}\right)$, que considera a retirada da tarefa $j$ de sua dupla sem que qualquer outra tarefa seja inserida nesta dupla. Ao custo deste arco é atribuído o valor $c\left(D\left[t_{j}\right] \backslash\left\{t_{j}\right\}\right)-c\left(D\left[t_{j}\right]\right) ;$

d. ligando cada dupla que recebe uma tarefa, sem a retirada de qualquer uma de suas tarefas originais, ao super-nó $\left(d_{k}, s\right)$, permitindo a formação de ciclos. Esse tipo de arco tem custo zero.

Nesse grafo são considerados somente os arcos descritos no item a) cuja substituição da tarefa $j$ pela tarefa $i$ resultar em uma dupla viável segundo as restrições impostas ao problema. O mesmo procedimento se aplica aos arcos descritos no item b). A Figura 1 mostra a construção da rede de melhoria para as duplas e as tarefas contidas na Tabela 1.

A rede $G(\boldsymbol{D})=(\boldsymbol{N}, \boldsymbol{E})$, gerada conforme descrito an-

Tabela 1. Conjunto de tarefas de três jornadas com partida e chegada no mesmo ponto

\begin{tabular}{|c|c|c|c|c|c|}
\hline \multicolumn{2}{|c|}{$<$ Dupla --- $1>$} & \multicolumn{2}{|c|}{ <Dupla --- $2>$} & \multicolumn{2}{|c|}{ < Dupla --- $3>$} \\
\hline T. Part. & Cheg. & T. Part. & Cheg. & T. Part. & Cheg. \\
\hline $104: 59$ & $05: 50$ & $\begin{array}{ll}2 & 05: 29 \\
4 & 07: 18 \\
6 & 08: 59\end{array}$ & $\begin{array}{l}06: 15 \\
08: 23 \\
10: 55\end{array}$ & $\begin{array}{ll}3 & 05: 45 \\
5 & 07: 14\end{array}$ & $\begin{array}{l}07: 01 \\
08: 05\end{array}$ \\
\hline
\end{tabular}

Legenda:

T: Tarefa; Part.: Partida; Cheg.: Chegada

teriormente, permite realizar movimento de realocação e troca 2-optimal, 3-optimal, ..., n-optimal. Os ciclos válidos encontrados nesta rede representam trocas que levam a uma melhoria no valor da função objetivo do problema.

Na Figura 1 , a troca cíclica $t_{4}-t_{5}-t_{4}$ corresponde à troca da tarefa 4 pela tarefa 5, e vice-versa, o que resulta em uma solução viável. Por outro lado, o ciclo $s$ $t_{4}-t_{5}-d_{T^{-}} S$ representa a substituição da tarefa 5 pela tarefa 4 na dupla 2 e da realocação da tarefa 5 da dupla 2 para a dupla 1 , troca esta que também resulta em uma solução viável. Uma troca cíclica do tipo $t_{1}-t_{2}-t_{3}-$ $t_{1}$ significa que a tarefa 1 substitui a tarefa 2 na dupla 3 , a tarefa 2 substitui a tarefa 3 na dupla 2, e a tarefa 3 substitui a tarefa 1 na dupla 1 . Caso uma troca deste tipo traga uma melhoria na função objetivo, tem-se um movimento do tipo 3-optimal.

\subsection{Identificando um ciclo válido}

Denomina-se um ciclo direcionado $W$ no grafo de melhoria $G(\boldsymbol{D})$ se as tarefas em $\boldsymbol{T}$ correspondentes aos nós em $W$ pertencem a diferentes duplas. Define-se um ciclo válido como um ciclo de custo negativo de um subconjunto desconexo em $G(\boldsymbol{D})$. Um

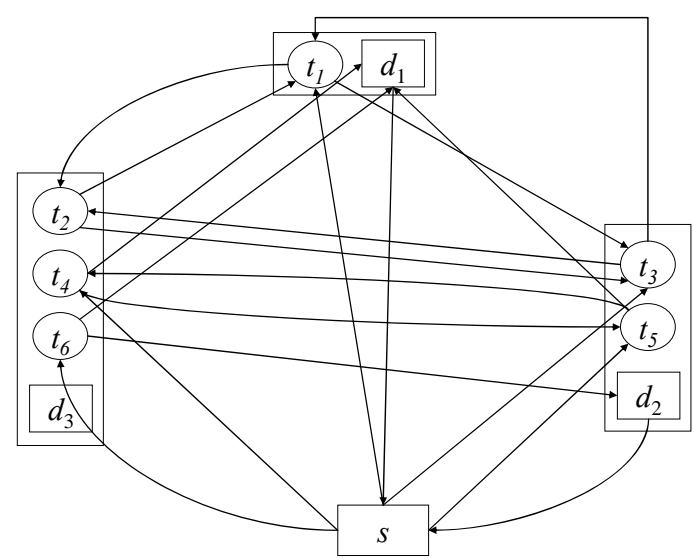

Figura 1. Rede de melhoria para as duplas e tarefas apresentadas na Tabela 1

ciclo válido no grafo $G(\boldsymbol{D})$ corresponde a uma troca cíclica, que leva a uma melhoria no valor da função objetivo do problema de particionamento descrito em (1). Esta é uma forma eficiente de realizar buscas por soluções que melhorem o valor objetivo na vizinhança de $\boldsymbol{V}(\boldsymbol{D})$. Portanto, é necessário encontrar ciclos váli- 
dos no grafo de melhorias $G(\boldsymbol{S})$. Diversos algoritmos são propostos na literatura para identificar ciclos válidos (Ahuja et al., 2001, 2003). Nos testes realizados pelos autores, as melhores heurísticas são capazes de identificar ciclos válidos em subconjuntos desconexos em redes com milhares de arcos, em tempos de processamento inferiores a um segundo (Ahuja et al., 2001).

Nesse trabalho foi utilizado o algoritmo do rotulamento modificado com a disciplina de fila para identificar um ciclo válido. Para implementar este algoritmo, é armazenado o número de vezes que cada nó é examinado. Se a rede não tiver ciclos válidos, cada nó será examinado no máximo $(n-1)$ vezes, onde $n$ é o número de nós da rede. Entretanto, se algum nó for examinado mais do que $(n-1)$ vezes, então a rede contém um ciclo. Para reconstruir o ciclo, basta partir do nó identificado e percorrer o vetor de nós predecessores até retornar ao nó de partida. Maiores detalhes podem ser obtidos em Ahuja et al. (1993).

\section{GRASP COM BUSCA EM VIZINHAÇA DE GRANDE PORTE PARA O PPT}

A heurística GRASP (Greedy Adaptive Search Procedure), proposta por Feo e Resende (1995), consiste de uma combinação de algoritmos construtivos para uma solução inicial com procedimentos de busca em uma vizinhança desta solução. Ela usa uma heurística construtiva gulosa com um dado grau de aleatoriedade para obter múltiplas soluções iniciais. A partir de cada solução, é aplicado um algoritmo de busca e escolhida a melhor solução local obtida pelo processo. Esta heurística permite implementações que podem diferir em função do processo de geração das soluções aleatórias e do método de busca local empregado. O GRASP básico para problemas de minimização é descrito pelo Algoritmo 1 (Figura 1).

Nesse algoritmo, é necessário definir como é realizada a construção randômica da solução e o método de busca a ser empregado, caracterizando assim a implementação GRASP para um dado problema. Tais definições são apresentadas a seguir. foi utilizada uma estratégia similar à prática adotada pelas empresas que alocam as duplas a um único veículo. Sendo assim, inicialmente não foi permitido que as duplas operassem em mais do que um veículo e o fator de aleatoriedade foi transferido para a quantidade de horas extras que cada tripulação poderia realizar. Então, o procedimento de construção da solução randômica gulosa para o PPT ficou com as seguintes características:

a. as tarefas de um dado veículo são ordenadas pelo horário de início e uma dupla é atribuída ao veículo recebendo a primeira tarefa do veículo;

b. a cada iteração, uma nova tarefa é atribuída à dupla corrente até que a dupla atinja uma certa quantidade de horas extras. A quantidade de horas extras permitidas a cada dupla é de $\alpha$ minutos sorteados aleatoriamente no intervalo $[0$, 120];

c. o procedimento se repete até que todos os veículos tenham suas tarefas atribuídas a alguma dupla.

As tarefas são alocadas às duplas se a jornada resultante estiver de acordo com as regras estabelecidas para o problema. Desta forma, todas as soluções randômicas são viáveis.

\subsection{Busca local}

A busca local é realizada pelo método de busca em vizinhança de grande porte, o BVGP. O método parte de uma solução viável para construir o respectivo grafo de melhoria associado ao problema. Posteriormente, é feita uma busca por um ciclo válido neste grafo. Ao encontrar um ciclo, são realizadas as trocas que ele representa e o grafo de melhoria é atualizado para que uma nova busca por um ciclo válido seja iniciada. Caso nenhum ciclo seja encontrado, o procedimento de busca é encerrado. O procedimento de busca é sintetizado no Algoritmo 2 (Figura 2), que foi incorporado ao GRASP descrito no Algoritmo 1, compondo assim o método proposto para a resolução do PPT.

\subsection{Solução randômica gulosa}

Para gerar uma solução randômica gulosa para o PPT,

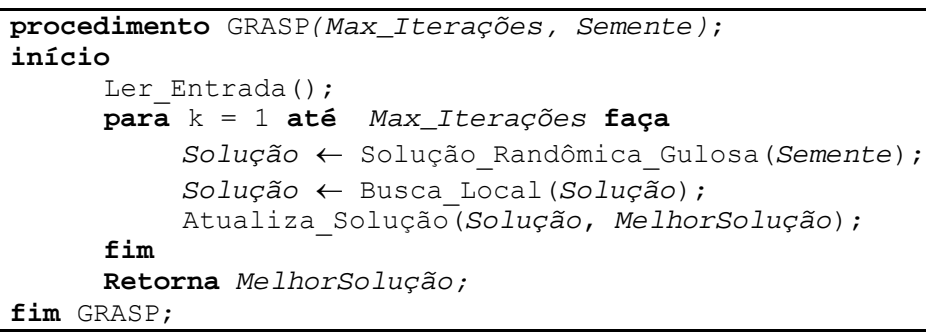

Figura 1. Algoritmo 1 - GRASP básico para um problema minimização 


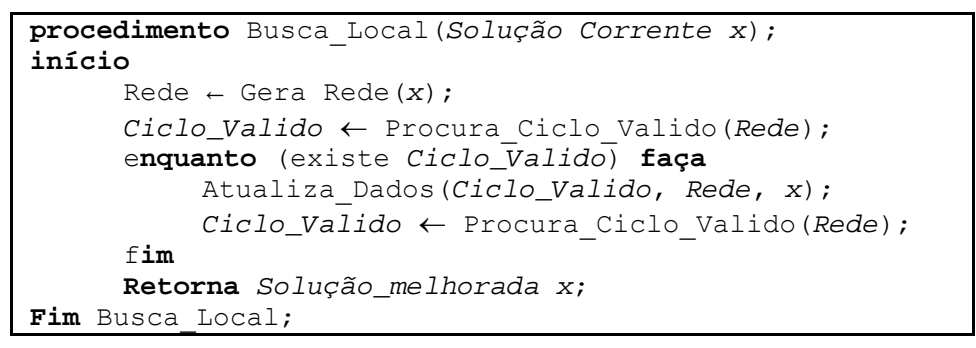

Figura 2. Algoritmo 2 - Busca em Vizinhança de Grande Porte

\subsection{Função de custo}

A função objetivo utilizada para avaliar uma solução do PPT tem as características apresentadas a seguir. Considere $D_{k}$ um subconjunto da partição $\boldsymbol{D}$ que representa as tarefas de uma dada dupla $k$. Então, o custo diário desta dupla é calculado pela expressão:

$$
c_{k}\left(D_{k}\right)=\text { Custo Fixo }+C_{1} \cdot H E\left(D_{k}\right)+C_{2} \cdot D P\left(D_{k}\right)
$$

Em que o Custo Fixo é o valor associado à manutenção de uma dupla no quadro de funcionários da empresa, $H E\left(D_{k}\right)$ é o tempo total de horas extras realizado pela dupla $k$ durante a sua jornada de trabalho e $D P\left(D_{k}\right)$ mostra se a dupla trabalha no regime de dupla pegada ou não. As constantes $C_{1}$ e $C_{2}$ são definidas pelo usuário, e são ajustadas de tal forma a adequar as soluções obtidas aos interesses operacionais da empresa. Neste modelo foi considerado um peso para as tripulações que realizam dupla pegada, como forma de controlar o número de jornadas deste tipo na solução. Este requisito se deve à prática das empresas do setor.

Embora os pesos adotados neste trabalho não reflitam fidedignamente os custos operacionais da escala, seus valores foram escolhidos tendo em vista uma série de trabalhos clássicos que adotam estratégia semelhante. Smith e Wren (1988), que abordam o PPT por meio de um modelo de recobrimento, utilizam uma rotina WAGES para calcular o custo de cada jornada, dado em função do tempo total de trabalhado. Este tempo total pode incluir horas extras, que são sobretaxadas. Desrochers e Soumis (1989) utilizam a constante HOURLY_RATE para o salário básico por hora de trabalho dos motoristas e a constante OVERTIME_RATE para as horas extras contidas na jornada. Na seção dos testes realizados com o modelo de geração de colunas, proposto pelos autores, é utilizado um fator de $50 \%$ de acréscimo das horas extras em relação à remuneração normal, entretanto os autores não apresentam o valor do salário básico dos motoristas. Shen e Kwan (2001) utilizam a expressão $f\left(D_{i}\right)+5.000$ onde $f\left(D_{i}\right)<<5.000$ e $f\left(D_{i}\right)$ fornece o custo da jornada $D_{i}$, que depende da empresa operadora. Os autores justificam a utilização do peso 5.000 como forma de reduzir o número de motoristas na heurística baseada em Busca Tabu.

É importante destacar que a estrutura de pesos ado- tada proporciona flexibilidade para ponderação das parcelas de custo de acordo com as características desejáveis para a solução, em termos de dupla pegada, horas extras, entre outras que podem ser incluídas na função de custo, definida em conjunto com a empresa operadora e de acordo com a realidade local. Por exemplo, caso a empresa julgue importante reduzir o número jornadas do tipo dupla pegada esta parcela receberá um peso maior na função de custo. Esse tipo de abordagem é fundamental para permitir a geração de programações de tripulantes que sejam consideradas viáveis pelos operadores, em especial para aqueles atributos que não são diretamente quantificáveis em termos de custos monetários, tais como dupla pegada e trocas de veículo.

\section{RESOLUÇÃO DE UM CASO REAL DE PROGRAMAÇÃO DAS TRIPULAÇÕES}

As heurísticas implementadas foram testadas com um conjunto de dados de uma empresa de transporte público que opera em Belo Horizonte, e se referem a uma semana do mês de março de 2009. Uma vez que o PPT não pode ser resolvido até a otimalidade, para problemas de grande porte, caso dos problemas estudados, os resultados obtidos pelas heurísticas foram comparados com as soluções adotadas pela empresa. Este tipo de comparação é praticamente inédito no Brasil para o setor de transporte público, pois as empresas raramente dispõem ou concordam em disponibilizar seus dados. A resistência se deve ao fato de que muitas empresas não cumprem as leis e acordos trabalhistas.

Os dados fornecidos pela empresa passaram por um procedimento de conferência, para que informações inconsistentes fossem eliminadas. Os casos que apresentavam problema de correlação entre veículos e duplas, e vice-versa, tiveram seus dados eliminados do estudo. Desta forma, pode ser observado na Tabela 2, que apresenta os dados dos problemas, que o número de viagens e de duplas apresentam uma variação significativa entre os dias da semana, diferentemente do que ocorre na prática. 
Tabela 2. Dados da escala de mão de obra operada pela empresa

\begin{tabular}{lrrrrrrr}
\hline \multicolumn{1}{c}{ SOLUÇ̃̃O DA EMPRESA } \\
\hline & Segunda & Terça & Quarta & Quinta & Sexta & Sábado & Domingo \\
\hline Horas Extra & $86: 41$ & $85: 55$ & $106: 05$ & $120: 14$ & $108: 11$ & $54: 35$ & $26: 57$ \\
Duplas Pegadas & 6 & 3 & 5 & 4 & 1 & 0 & 0 \\
Total de Duplas & 134 & 130 & 149 & 162 & 155 & 124 & 68 \\
Total de viagens & 772 & 730 & 883 & 945 & 874 & 704 & 392 \\
\hline FO & 1.364 .404 & 1.322 .420 & 1.518 .460 & 1.651 .256 & 1.576 .564 & 1.253 .100 & 686.468 \\
\hline
\end{tabular}

O custo de uma solução do PPT, descrito da equação (2), foi avaliado considerando os seguintes valores para os parâmetros: Custo Fixo $=10.000$, coeficiente de horas extras $C_{l}=4$ e coeficiente de duplas pegadas $C_{2}=600$. Dessa forma, o custo da tripulação tem um valor elevado para que a redução no número de duplas seja o principal critério de otimização, seguindo-se pela redução de dupla pegada e finalmente, das horas extras.

O valor da FO adotada pela empresa foi calculado aplicando os coeficientes definidos para o modelo aos seus respectivos dados operacionais. Este valor foi utilizado como base de comparação e análise da qualidade destas soluções.

Tendo em vista o caráter randômico da metaheurística GRASP, foram realizadas três rodadas diferentes da heurística para cada problema abordado, cada uma com tempo de processamento de 45 minutos. Nestes testes, o modelo foi parametrizado para gerar soluções compatíveis com as soluções adotadas pela empresa. Ou seja, foram estudados três cenários, variando o número máximo de trocas de veículos que cada dupla pode realizar durante a operação. Uma dupla só pode trocar de veículo se ambos os veículos estiverem no mesmo ponto no momento da troca. Esta é uma particularidade da operação da empresa, que não permite que uma dupla se desloque de um ponto para outro, na condição de passageiros, para dar continuidade à sua jornada de trabalho em outro veículo. No caso de dupla pegada, pode haver a troca de pontos desde que ela ocorra entre uma pegada e outra, quando o intervalo de tempo é superior a duas horas.

\subsection{Resultados obtidos}

Os resultados das Tabelas 3 a 5 correspondem à resolução dos mesmos problemas de geração de escala en- frentados pela empresa. Na resolução destes problemas, os dados de entrada correspondem à programação dos veículos realizada pela empresa entre os dias 2 e 8 de março de 2009, desconsiderados os dados inconsistentes.

A Tabela 3 contém informações das soluções geradas pela heurística GRASP, considerando que as duplas não podem realizar qualquer troca de veículo durante suas jornadas. Esta é a situação que mais se assemelha à operação da empresa, uma vez que as trocas de veículos, embora ocorram, são em pequeno número. Na última linha são informadas as porcentagens de melhoria/ganho obtidas em relação às soluções da empresa, conforme os pesos adotados neste trabalho. O modelo permite ao usuário realizar um refinamento da solução em função da variação dos valores atribuídos aos pesos da função de custo (2).

Os resultados apresentados na Tabela 4 foram obtidos considerando a regra que permite que cada dupla realize no máximo uma troca de veículo durante a jornada. A tendência é que as soluções melhorem na medida em que o número de trocas aumenta, pois a flexibilização da operação permite um melhor aproveitamento da mão de obra. Este resultado é observado em todos os dias, com exceção da programação do sábado, quando há um ligeiro aumento na FO em relação ao cenário anterior.

Finalmente, considerando que as duplas podem realizar no máximo 2 trocas de veículos durante suas jornadas, foram obtidas as soluções que constam da Tabela 5. Neste último cenário, as exceções observadas quanto à tendência de melhoria da FO com o aumento nas trocas de veículos são verificadas nos problemas de terça, sábado e domingo.

Tabela 3. Solução GRASP-S com o número de troca veículo/tripulação igual a 0

\begin{tabular}{lrrrrrrr}
\hline & Segunda & Terça & Quarta & Quinta & Sexta & Sábado & Dom \\
\hline Horas Extras & $76: 29$ & $54: 41$ & $76: 23$ & $86: 20$ & $66: 38$ & $57: 52$ & $22: 04$ \\
Duplas Pegadas & 16 & 8 & 11 & 16 & 11 & 17 & 5 \\
Total de Duplas & 124 & 125 & 144 & 155 & 149 & 110 & 62 \\
Trocas de Veics. & 0 & 0 & 0 & 0 & 0 & 0 & 0 \\
\hline FO & 1.267 .956 & 1.267 .924 & 1.464 .932 & 1.580 .320 & 1.512 .592 & 1.124 .088 & 628.296 \\
$\%$ Melhora & $7,07 \%$ & $4,12 \%$ & $3,53 \%$ & $4,30 \%$ & $4,06 \%$ & $10,30 \%$ & $8,47 \%$ \\
\hline
\end{tabular}


Tabela 4. Solução GRASP-S permitindo no máximo uma troca veículo/tripulação

\begin{tabular}{lrrrrrrr}
\hline & Segunda & Terça & Quarta & Quinta & Sexta & Sábado & Domingo \\
\hline Horas Extras & $68: 29$ & $48: 40$ & $65: 46$ & $71: 45$ & $60: 30$ & $51: 08$ & $27: 09$ \\
Duplas Pegadas & 14 & 7 & 11 & 12 & 10 & 15 & 7 \\
Total de Duplas & 124 & 125 & 144 & 154 & 149 & 111 & 58 \\
Trocas de Veics. & 45 & 31 & 48 & 54 & 38 & 20 & 6 \\
\hline FO & 1.264 .836 & 1.265 .880 & 1.462 .384 & 1.564 .420 & 1.510 .520 & 1.131 .272 & 590.716 \\
\% Melhora & $7,30 \%$ & $4,28 \%$ & $3,69 \%$ & $5,26 \%$ & $4,19 \%$ & $9,72 \%$ & $13,95 \%$ \\
\hline
\end{tabular}

Tabela 5. Solução GRASP-S permitindo no máximo duas trocas veículo/tripulação

\begin{tabular}{|c|c|c|c|c|c|c|c|}
\hline & Segunda & Terça & Quarta & Quinta & Sexta & Sábado & Domingo \\
\hline Horas Extras & $66: 32$ & $48: 49$ & $83: 13$ & $70: 33$ & $56: 11$ & $55: 57$ & $26: 05$ \\
\hline Duplas Pegadas & 14 & 7 & 12 & 11 & 9 & 14 & 8 \\
\hline Total de Duplas & 124 & 125 & 143 & 154 & 149 & 111 & 58 \\
\hline Trocas de Veícs. & 37 & 59 & 45 & 93 & 90 & 26 & 14 \\
\hline $\mathrm{FO}$ & 1.264 .368 & 1.265 .916 & 1.457 .172 & 1.563 .532 & 1.508 .884 & 1.131 .828 & 591.060 \\
\hline \% Melhora & $7,33 \%$ & $4,27 \%$ & $4,04 \%$ & $5,31 \%$ & $4,29 \%$ & $9,68 \%$ & $13,90 \%$ \\
\hline
\end{tabular}

\subsection{Discussão dos resultados obtidos}

Com base nos resultado apresentados nas tabelas acima, observa-se que, em geral, as soluções melhoram na medida em que aumenta o número permitido de trocas de veículo realizadas pelas tripulações. Entretanto, exigir que uma tripulação faça duas trocas durante uma jornada diária de trabalho é uma situação que não ocorre no dia-a-dia. O cenário de grande interesse prático, manifestado pela empresa, é o caso em que as duplas realizam até uma troca de veículo durante a operação. Esta é uma prática considerada viável, pois é uma forma de diversificar as atividades da dupla sem incorrer em uma ruptura muito grande com a prática atual. Assim, analisando os melhores resultados para este cenário e considerando os dias úteis, que são aqueles que determinam o número de duplas e a folha de pagamento da empresa, observa-se uma redução variando entre $3,36 \%$ e $7,46 \%$ no número de duplas, acompanhado de uma redução entre 20,93\% e 44,44\% no número de horas extras.

Além das trocas de veículos, um fator que diferencia as soluções obtidas pelo método das soluções da empresa é o número de duplas pegadas. Nas soluções da empresa ocorrem no máximo 6 duplas pegadas contra 16 geradas pela heurística, nas soluções referentes aos dias úteis. Embora o aumento seja maior do que $100 \%$, em valores absolutos, não há um comprometimento operacional das soluções.

\section{CONCLUSÕES}

Neste trabalho foi apresentada uma utilização inédita da técnica de Busca em Vizinhança de Grande Porte (Very Large-Scale Neighborhood Search) para resolver o Problema de Programação de Tripulações (PPT) de empresas que atuam no sistema de transporte pú- blico. A técnica de busca foi combinação com a heurística GRASP, permitindo obter soluções significativamente melhores do que aquelas adotadas pela empresa, e com tempos de processamento não elevados.

Para aplicar a técnica de busca, foi concebida uma rede de melhoria para o PPT. Tal rede permite melhorar a qualidade das soluções por meio da detecção de ciclos válidos. Uma vez encontrado um ciclo desta natureza, a solução corrente sofre necessariamente uma melhoria. A melhoria é alcançada com a realização das realocações e trocas inerentes ao ciclo.

A técnica de BVGP permite realizar não só movimentos de realocação e troca de viagens, mas também movimentos 3-optimal, 4-optimal, ..., n-optimal, onde $n$ corresponde ao número de tripulações na solução corrente. Desta forma, explora-se um espaço de busca muito mais complexo do que aqueles pesquisados na maioria das heurísticas implementadas para os problemas, que contam apenas com movimentos de realocação e troca de tarefas. O tempo de busca na vizinhança é minimizado com a construção do grafo de melhoria e a utilização de algoritmos de fluxo em redes para encontrar ciclos negativos.

A heurística foi testada com dados operacionais recentes de uma empresa que atua em Belo Horizonte, o que o permitiu constatar que o modelo é capaz de produzir soluções com as mesmas características daquelas utilizadas na prática. Além disso, as soluções geradas pela heurística permitiram um ganho considerável tendo em vista o número de duplas empregadas e a quantidade de horas extras contidas na escala diária da empresa. A heurística ainda é capaz de gerar soluções considerando diferentes cenários operacionais, os quais ainda não são adotados pelas empresas que operam no Brasil.

A principal conclusão deste trabalho é que a heurística GRASP, associada à técnica de Busca em Vizi- 
nhança de Grande Porte, se aplica perfeitamente à resolução do problema de programação de tripulações no sistema de transporte público. Os resultados são promissores e a capacidade de modelar problemas práticos não deixa dúvida quanto ao seu potencial de aplicação real do modelo heurístico.

O trabalho abre uma série de possibilidades para explorar diferentes heurísticas, pois foi observado que o resultado de uma busca é fortemente dependente da solução inicial. Sendo assim, uma continuidade desse trabalho consiste em combinar a técnica de busca com outras heurísticas construtivas que, periodicamente, realizam perturbações na solução corrente através de movimentos diversos.

\section{AGRADECIMENTOS}

Os autores agradecem ao CNPq, à FAPEMIG e à BHTRANS pelo apoio recebido durante o desenvolvimento deste trabalho.

\section{REFERÊNCIAS BIBLIOGRÁFICAS}

Ahuja, R. K.; O. Ergun; J. B. Orlin e A. P. Punnen (2002) A survey of very large scale neighborhood search techniques. Discrete Applied Mathematics, v.123., p. 75-102.

Ahuja, R. K.; T. L. Magnanti e J. B. Orlin (1993). Network Flows: Theory, Algorithms, and Applications. Prentice Hall, N. J.

Ahuja, R. K.; J. B. Orlin e D. Sharma (2000) Very large scale neighborhood search. International Transactions in Operations Research v.7, p. 301-317.

Ahuja, R. K.; J. B. Orlin e D. Sharma (2001) Multi-exchange neighborhood search algorithms for the capacitated minimum spanning tree problem. Mathematical Programming v. 91, p.71-97.

Ahuja, R. K.; J. B. Orlin e D. Sharma (2003) A composite very large-scale neighborhood structure for the capacitated minimum spanning tree problem . Operations Research Letters, v. 31.3, p. 185-194.

Barnhart, C.; E. L. Johnson; G. L. Nemhauser; M. P. Savelsbergh e P. H. Vance (1998) Branch-and-price: column generation for solving huge integer programs. Operations Research, v.46, p.316-329.

Clement, R. e A. Wren (1995) Greedy Genetic Algorithms, Optimizing Mutantis and Bus Driver Scheduling. Computer-Aided Transit Scheduling, p. 213-235, Springer-Verlag.

Desrochers, M. e F. Soumis (1989) A Column Generation approach to the urban transit crew scheduling problem. Transportation Science, v. 23, p. 1-13.

Desrochers, M; J. Gilbert; M. Sauve e F. Soumis (1992) CREW-OPT: Subproblem modeling in a column generation approach to urban crew scheduling. Computer-Aided Transit Scheduling, Springer, p. 395-406.

Feo, T.A. e M. G. C. Resende (1995). Greedy randomized adaptive search procedures, Journal of Global Optimization, v.6, p. 109-133.

Fores, S.; L. Proll e A. Wren (1999) An Improved ILP System For Driver Scheduling. Computer-Aided Transit Scheduling, Wilson, N. H. M. (ed.), Springer, Berlin , p. 43-61.

Freling, R.; D. Huisman e A. Wagelmans (2001) Applying an Integrated Approach to Vehicle and Crew Scheduling in Practice. S. Voss e J. Daduna (eds.), Computer-Aided Scheduling of Public Transport, Lecture Notes in Economics and Mathematical Systems, Vol. 505, p. 73-90. Springer.

Friberg, C. e K. Haase (1999) An exact branch and cut algorithm for the vehicle and crew scheduling problem. Computer-Aided Transit Scheduling, Wilson, N. H. M. (ed.), Springer, Berlin, p. 63-80.

Lourenço, H. R.; J. P. Paixão e R. Portugal (2001) Multiobjective metaheuristics for the bus-driver scheduling problem. Tranportation Science, v. 35, p. 331-343.

Marinho, E. H.; M. J. F. Souza e G. P. Silva (2004) Busca Tabu Aplicada ao Problema de Programação de Tripulações de Ônibus Urbano. In: Simpósio Brasileiro de Pesquisa Operacional, XXXVI, p. 1471-1482.
Shen, Y. e R. S. K. Kwan (2001) Tabu Search for Driver Scheduling. In: Daduna, J. R.; Voss, S. (Ed.). Computer-Aided Scheduling of Public Transport. Berlim: Springer-Verlag, p. 121-135.

Silva, G. P.; M. J. F. Souza e J. Atzingen (2007) Análise Comparativa de Métodos para Resolver o Problema de Programação de Tripulações. Revista Produção OnLine v.7, n. 2, p. 126-143.

Silva, G. P.; M. J. F. Souza e J. M. C. B. Alves (2002) Resolução do Problema de Programação Diária da Tripulação de Ônibus Urbano via Simulated Annealing, XVI Congresso de Pesquisa e Ensino em Transportes, Panorama Nacional de Pesquisa em Transportes, vol. 2, pp. 95-104, ANPET.

Silver, E. A. (2004). An overview of heuristic solution methods. Journal of the Operational Research Society v. 55, n.9, p.936-956.

Smith, B. M. e A. Wren (1988) A Bus Crew Scheduling System Using a Set Covering Formulation. Transportation Research, v. 22A, p. 97-108.

Souza, M. J. F; L. X. T. Cardoso; G. P. Silva; M. M. S. Rodrigues e S. M. S. Mapa (2004) Metaheurísticas aplicadas ao Problema de Programação de Tripulações no Sistema de Transporte Público, Tendências em Matemática Aplicada e Computacional, v. 5, 12 p. 357-368.

Souza, M. J. F; G. P. Silva e S. M. S. Mapa (2004) Métodos de Pesquisa em Vizinhança Variável Aplicados à Resolução do Problema de Programação Diária de Tripulação de Ônibus Urbano. XVIII Congresso de Pesquisa e Ensino em Transportes, Panorama Nacional de Pesquisa em Transportes, v. 2. p. 1492-1502.

Wren, A.; R. S. K. Kwan e A. S. K. Kwan (2000) Hybrid genetic algorithms for scheduling bus and train drivers. Proceedings of the 2000 Congress on Evolutionary Computation CEC 00, p. 285292.

Wren, A. e D. O. Wren (1995) A Genetic Algorithm for Public Transport Driver Scheduling, Computer and Operations Research, v. 22, p. 101-110 